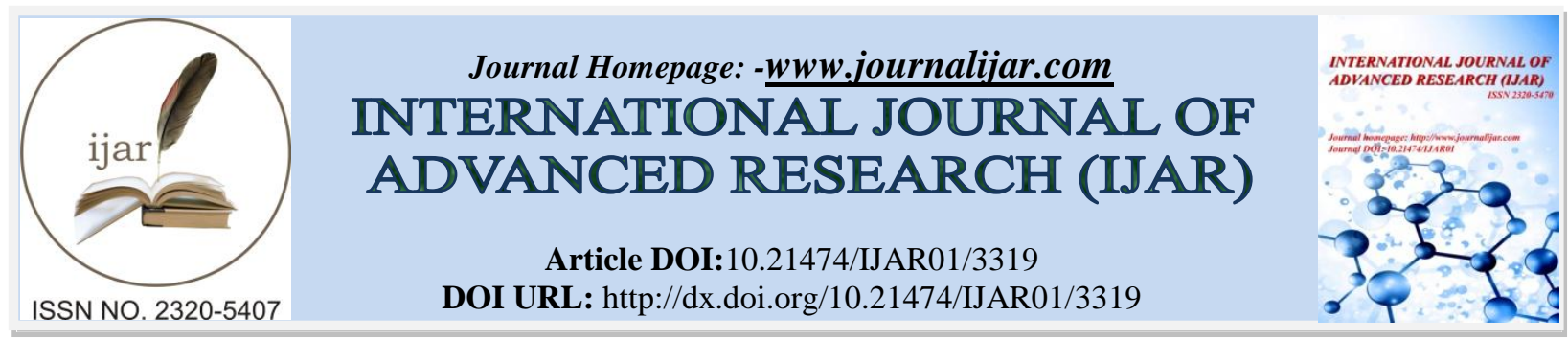

RESEARCH ARTICLE

\title{
CAN WE STILL RELY ON ABDOMINAL X-RAY IN DIAGNOSING BOWEL OBSTRUCTION?
}

\author{
Maha M. Alsharif ${ }^{1}$, Azza A. Azzouz ${ }^{1}$, Mohammad Wazzan ${ }^{2}$, Ahmed H Abduljabbar ${ }^{2}$ and Ahmad S Mufti' \\ 1. Medical intern - King Abdulaziz University - Jeddah - Saudi arabia. \\ 2. Assistant professor, abdominal imaging radiologist, Deputy chairman of radiology department, Head of \\ abdominal imaging, King Abdulaziz University - Jeddah - Saudi Arabia.
}

\section{Manuscript Info}

Manuscript History

Received: 21 December 2016

Final Accepted: 25 January 2017

Published: February 2017

Key words:-

X-ray, CT scan, bowel obstruction.

\section{Abstract}

Background: Bowel obstruction (BO) describes as "failure of passage of intestinal contents", it's common surgical emergency and a major cause of morbidity, during last twenty years there was a huge development in radiological techniques used to diagnose bowel obstruction. This study aimed to compare reliability between abdominal plain radiography and CT for detection of suspected cases.

Method:A retrospective study including 261 patients suspected SBO who underwent CT scan and abdominal x-ray in king Abdulaziz University hospital, during the period from May 2016 to November 2016.

Results:From 261 patients suspected BO,119 (45.6\%) were male and $142(54.4 \%)$ female, with mean age score $56.3 \pm 17.7,132(50.8 \%)$ were presented as abdominaldistension, follow by 129 (49.6\%) abdominal pain, $69(26.5 \%)$ as vomiting , $61(23.4 \%)$ as constipation, and $11(4.2 \%)$ as diarrhea ,from the total number 78 (29.9\%) cases reported as positive intestinal obstruction by CT, where 154 (59.4\%) cases reported as suspected intestinal obstruction by $\mathrm{x}$-ray .

Conclusion:The early precise diagnosis of bowelobstruction helps in decreasing the morbidity and mortality rate. Plain abdomen radiography is suitable technique in the emergency cases and follow up tool after operation, however CT scan is the more accurate technique.

Copy Right, IJAR, 2017,. All rights reserved.

\section{Introduction:-}

Bowel obstruction (BO) describes as "failure of passage of intestinal contents",(1)it's common surgical emergencyand a major cause of morbidity which requires a correct diagnosis and rapid management .(2)It could be mechanical or paralytic in origin, it is classified in to two types; large and small intestinal obstruction where 60$80 \%$ of the cases had small bowel which is mechanical in nature in the majority of the cases. $(3,4)$

A multidisciplinary approach includes; familiarity of pathophysiology, clinical skills and the accurate imaging of choice are needed to reach the correct diagnosis, (5)majority of bowel obstruction cases diagnosed based on plain radiography and it has been the initial diagnostic test since it characterized by fast accessibility, easily to perform and low priced more over it has specific key features of BO. Although plain X-ray is the initial test, CT scan is the gold standard in diagnosing BO and added the advantage of detectthe site and the cause of obstruction. Since imaging has a crucial role in management plan, the purpose of the study is to compare the reliability between 
abdominal plain radiography and CT for detection thesuspected cases clinically with obstruction, and to evaluate optimal radiological modality or identifying new criteria for early assessment, hence allow fast intervention. ( $1,3,4,5,6)$.

\section{Material and Methods:-}

A retrospective study including 261patients suspected SBO who underwent CT scan and abdominal x-ray in king Abdulaziz University hospital. Comparing CT scan and abdominal x-ray with a maximum time of 72 hours between each one for the accuracy of diagnosis. We reviewed the electronic files from hospital medical record. Data sheet items included personal data (age, gender, MRN), X-ray imagine had been reviewed for detection of 3 specific signs for each radiograph which were; air fluid number, paucity of gases and diameter of the largest bowel, where diameters guided by 3/6/9 rule. Each CT scan reviewed and confirmed by reports with focusing on: contrast administration route, the presence of obstruction, its sites and complications if present. The most important question was if there is an obstruction by CT, all specific signs of both CT and radiograph were taken. If the CT was nondiagnostic for intestinal obstruction,only $\mathrm{x}$ ray specific signs were recorded.

\section{Result:-}

This study included 261 patients suspected SBO, of which 119 (45.6\%) were male and 142 (54.4\%) female, with mean age score 56.3 \pm 17.7 . (Table 1)

Table 1:-Demographic data.

\begin{tabular}{|c|c|c|}
\hline \multicolumn{2}{|c|}{ N } & \% \\
\hline Variables & Gender & 45.6 \\
\hline Male & 119 & 54.4 \\
\hline Female & 142 & Rang (min-max) \\
\hline Variables & Mean \pm SD & (17-90) \\
\hline Age & $\mathbf{5 6 . 3} \pm$ & \\
\hline
\end{tabular}

The clinical presentation and radiology findings ,132 (50.8\%) were presented as abdominaldistension , follow by $129(49.6 \%)$ abdominal pain, $69(26.5 \%)$ as vomiting , $61(23.4 \%)$ as constipation, 45 (17.3\%)as intestinal obstruction, and $11(4.2 \%)$ as diarrhea ,from the total number 78 (29.9\%) cases reported as positive intestinal obstruction by CT, where $154(59.4 \%)$ cases reported as suspected intestinal obstruction by x-ray (Table 2)

Table 2:-Type of presentation:

\begin{tabular}{|c|c|c|}
\hline Variables & $\mathbf{N}$ & $\%$ \\
\hline \multicolumn{3}{|c|}{ Type } \\
\hline Abdominal pain & 129 & 49.6 \\
\hline Abdominal distension & 132 & 50.8 \\
\hline Vomiting & 69 & 26.5 \\
\hline Diarrhea & 11 & 4.2 \\
\hline Constipation & 61 & 23.4 \\
\hline Fever & 2 & 0.8 \\
\hline \multicolumn{3}{|c|}{ CT findings ( intestinal obstruction on CT) } \\
\hline Negative & 182 & 69.7 \\
\hline Positive & 78 & 29.9 \\
\hline \multicolumn{3}{|c|}{ X-ray performing ( there is $\mathrm{x}$-ray for this patient or not) } \\
\hline No & 106 & 40.6 \\
\hline Yes & 154 & 59.4 \\
\hline
\end{tabular}

CT findings includes, 71 (27.2\%) cases received oral cont, while 67 (257\%) received IV contrast, and only 11(3.4\%) cases received rectal. More than third (29 patients $37.2 \%$ ) reported Ilium as the site of obstruction, followed by Jejunum (21 patients $-26.9 \%$ ) and colon (21 patients-26.9\%), also more than third (28 patients $-35.9 \%)$ reported mechanical obstruction as the main cause and on the other hand 70 patients $(89.9 \%)$ cases reported no complication.

(Table 3). 
Table 3:- CT findings.

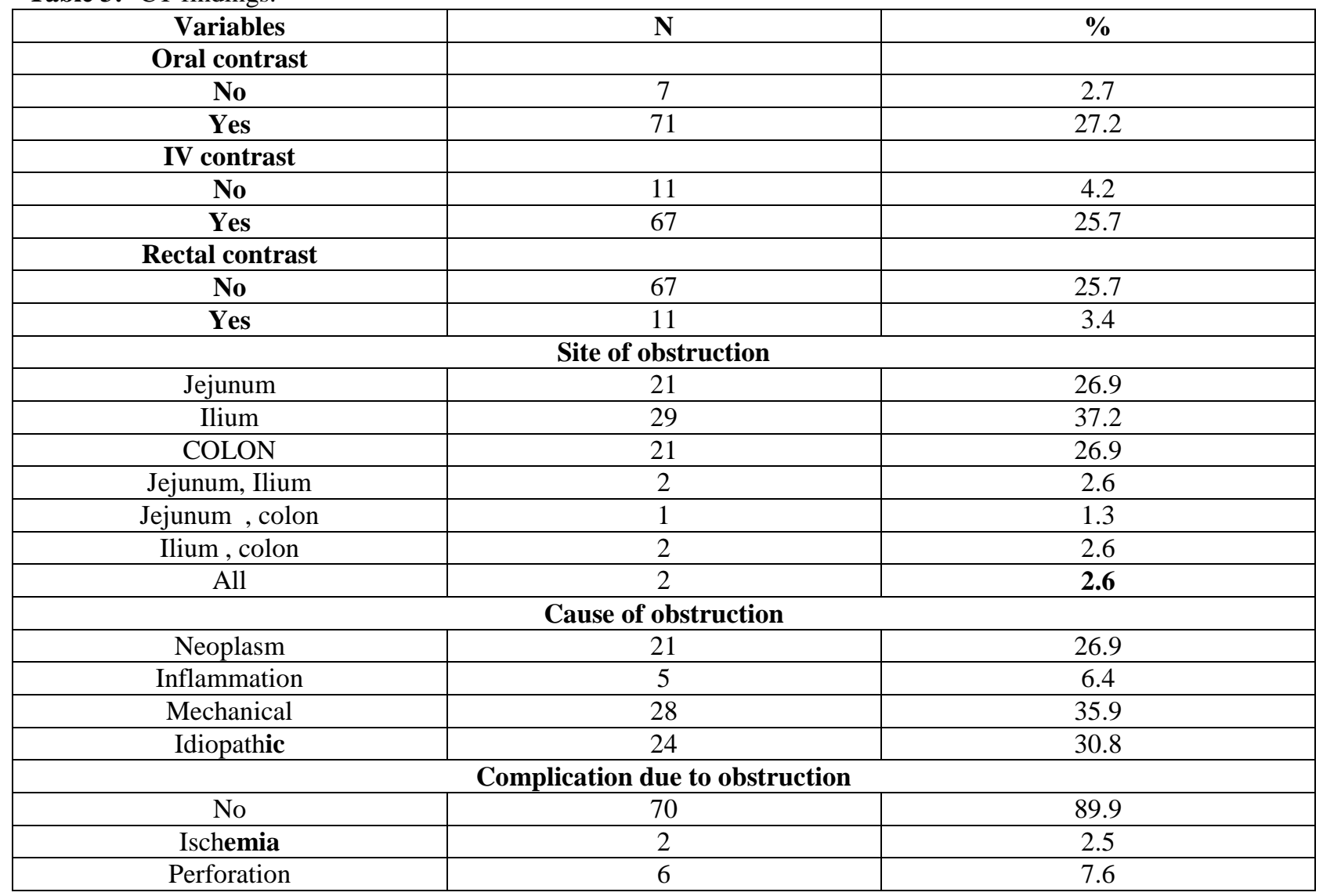

The x-ray findings showed the mean score of diameters was $4.9 \pm 1.7$ rang (2.4-10.0) where $44.8 \%$ of the cases reported diameters measurement between 4.0-6.0, the median score of air/fluid levels was 4 (0-6) where 19 (28.4\%) cases reported air fluid level between 5.0-9.0and 10 (13.9\%) between 3.0-4.0. More than two third of the patients (107 cases $-69 \%)$ reported no paucity bowel gases followed by 25 patients $(16.1 \%)$ cases in all over the abdomen. (Table 4).

Table 4:-X-ray findings.

\begin{tabular}{|c|c|c|}
\hline Variables & $\mathbf{N}(67)$ & $\%$ \\
\hline \multicolumn{3}{|c|}{ Paucity } \\
\hline No & 42 & 61.0 \\
\hline RUQ & 3 & 5.0 \\
\hline $\mathrm{RS}$ & 4 & 6.0 \\
\hline MS & 1 & 1.5 \\
\hline $\mathrm{LS}$ & 1 & 1.5 \\
\hline RLQ & 1 & 1.5 \\
\hline LLQ & 2 & 3.0 \\
\hline All over & 12 & 16.0 \\
\hline RUQ, MS, RLQ & 2 & 3.0 \\
\hline RLQ,MLQ,LLQ & 1 & 1.5 \\
\hline \multicolumn{3}{|c|}{ Diameters } \\
\hline $2.5-3.9$ & 20 & 29.9 \\
\hline 4.0-4.9 & 15 & 22.4 \\
\hline $5.0-5.9$ & 15 & 22.4 \\
\hline 6.0-6.9 & 9 & 13.4 \\
\hline $7.0-10.0$ & 8 & 11.9 \\
\hline Mean \pm SD & \multicolumn{2}{|c|}{$4.9 \pm 1.7$} \\
\hline
\end{tabular}




\begin{tabular}{|c|c|c|}
\hline \multicolumn{2}{|c|}{ Air/fluid } \\
\hline Rang (min-mx) & 29 & 43.3 \\
\hline$\leq \mathbf{2 . 0}$ & 10 & 14.9 \\
\hline $\mathbf{3 . 0 - 4 . 0}$ & 19 & 28.4 \\
\hline $\mathbf{5 . 0 - 9 . 0}$ & 9 & 13.4 \\
\hline $\mathbf{1 0 . 0 - 1 4 . 0}$ & \multicolumn{2}{|c|}{4} \\
\hline Median & \multicolumn{2}{|c|}{$(0-6)$} \\
\hline Quartile (25-75) & \multicolumn{2}{|c|}{} \\
\hline
\end{tabular}

The results showed significant difference between CT findings and $\mathrm{x}$-ray findings, where from the 154 cases suspected by obstruction 68(44.1\%) cases were positive in CT, while from the 106 cases negative in $\mathrm{x}$-ray 10 cases were positive in CT $(\mathrm{p}<0.0001)$. (Table 5)

Table 5:- Comparing between CT scan and X-ray findings:

\begin{tabular}{|l|l|l|l|l|}
\hline \multicolumn{2}{|l|}{ Variables } & \multicolumn{2}{|l|}{ CT findings } & \multirow{2}{*}{ P value } \\
\cline { 3 - 4 } \multicolumn{2}{|l|}{ Positive } & Negative & \\
\hline \multirow{2}{*}{ X-ray } & Yes & $\mathbf{6 8}(87.0 \%)$ & $\mathbf{8 6}(47.3 \%)$ & \multirow{2}{*0.0001*}{} \\
\cline { 2 - 4 } & No & $\mathbf{1 0}(\mathbf{1 3 . 0 \%})$ & $\mathbf{9 6 ( 5 2 . 7 \% )}$ & \\
\hline
\end{tabular}

\section{Discussion:-}

\section{Bowel obstruction:-}

Bowel obstruction is a critical clinical condition and responsible of $20 \%$ of surgical intervention due to acute abdominal condition. (4)

The current study results showed that the most common symptoms was abdominal pain , abdomen distension and constipation, this consistent with the results of previous study, $(\mathbf{1 , 4 , 7})$ The diagnosis of BO is a real challenging where it has variety in symptoms and signs regarding the nature of presentation (acute or subacute), complete obstruction or partial, and if it is simple or there is complication such as strangulation or perforation. $(\mathbf{3 , 5})$

\section{Radiology modalities:-}

During last twenty years, there was a huge development in radiological techniques used to diagnose $\mathrm{BO}$, where the most important step is to choose the most suitable method to detect the medical condition and provide the correct diagnosis, $(\mathbf{3 , 5})$ The current study focused on comparing between abdominal plain radiography and $\mathrm{CT}$ for detection BO, where from 260 patients suspected patients with BO, only 68 patients diagnosed with BO and confirmed by CT $\&$ abdominal plain radiography , 86 cases were suspected by radiograph and negative in CT, while 10 cases showed normal finding in radiograph but they were diagnosed as BO by CT , similar results were found in others studies where the authors reported that CT scan comparing with has higher sensitivity (93\%), specify (100\%) \&accuracy ( $93 \%$ )while plain abdominal radiography could misdiagnosis $20 \%$ of the cases.(1,2,3,4)

\section{Abdominal plain radiography:-}

Plain abdominal radiography was the first radiology technique used with physical examination to diagnose BO since $1920,(\mathbf{5})$ with reliability level between $46-86 \%,(\mathbf{1 , 5}, \mathbf{6})$ this disparity in reliability between studies was due to several factors such as study design, picking participants , use of the term "non-specific bowel gas pattern,", involved patients with recent abdomen surgery,(6)the result of current study consistent with previous study where $44 \%$ of the cases were diagnose by plain abdominal radiography.

Several studies conduct to evaluate theuse of plain abdominal radiography asdiagnostic tool of BO. These studies demonstrated the use of plain abdominal radiography as the primary evaluation tool in detecting $\mathrm{BO}$ among suspicion cases presented to ER ,(1,6,8)where the advantages of this technique are :lower cost, availability, easy access, high sensitivity in detecting high grade obstruction and the ability of using it to follow the progress after surgical intervention. $(\mathbf{1 , 4 , 6 , 9 , 1 0 )}$, The main signs of $\mathrm{BO}$ in on abdominal radiographs were " distended small bowelloops of greater than $3 \mathrm{~cm}$, paucity of air in the large bowel, collapsed colon, differential air-fluid levels, and thickened bowel wall",(1,5), the results of the current study agreed with previous study where more than tow third showed dilated more than $3 \mathrm{~cm}$, more than half showed air fluid level longer than $2.5 \mathrm{~cm}$ and all 67 suspected cases with $\mathrm{BO}$ and performedplain abdominal radiography showed paucity abdomen , 
On the other hand several studies reported that performed plain abdominal is confusing \& uncertain in detecting BO (5) where a few limitations were recorded on this tool: unable to detect cause unless in inguinal hernias, and gallstone ileus $\mathbf{( 5 , 1 1 )}$, the imagine could be normal in early obstruction and in low grade obstruction, $(\mathbf{1 , 4 , 1 2})$ these limitations made researchers emphasize on the need to perform CT scan in the cases of high suspicion of BO even with negativeradiography imagine. (1)

\section{Computed tomography (CT):-}

In1979 CT scan was invention, since then it became one of the important radiology technique in diagnosis of BO.(5)CT scan could be categorized toroutine abdominal CT, CT enteroclysis (CTEc), and CT enterography (CTE). (6), Routine abdominal CT is the most commonmethod using in diagnosis of BO because it is fast and could be run without oral contrast where "the retained intraluminal fluid serves as a natural negative contrast agent"this mean it could be used with patients suffered from nausea and vomiting without fearing from contrast's aspiration . (3,6) however in the cases of partial obstruction oral contrast need to be given and in low grade partial obstruction CTE is preferred more than routine CT because it is provide better evaluation to $\mathrm{BO}$. $(\mathbf{3 , 6 , 1 3 )}$

There are five major questions in BO cases where CT provide doctors with the answer of them which make it a valuable tool in diagnosis of BO: (Is there an obstruction in the bowel?, Where is the transition point (TP)?What is the cause?, What is the severity degree ?,Is it simple or complicated?). (2,4)

Bowel obstruction diagnosed by CT when "the small bowel is dilated to a caliber of more than $2.5 \mathrm{~cm}$ with a distinct point of transition and normal caliber bowel beyond". $(\mathbf{5 , 1 4})$

Regarding the question of transition point (TP), it is look like a beak and specified by "identifying a caliber change between the dilated proximal and collapsed distal small bowel loops".(4,5,11,15)

Concerning the question of the cause of obstruction, The main causes of BO in this study were mechanical, neoplasm, and idiopathic, where the evaluation of transition point is essential to identify the real cause , (3)similar results were found in India study the main cause were Intestinal adhesion and tumor (1269), and in Pakistan study were adhesions 29 (40\%), neoplasm $12(17 \%)$ and hernias $7(10 \%)$. (2970), where adhesions reported mostly in patients with history of previous abdomen surgery and it responsible of 60-80\% from small BO cases. (1,16)

Determination of severity degree of BO either it is complete or partial rely on 3 factors: " distal collapse, proximal bowel dilatation, and the transit of contrast", (4)If the oral contrast material pass through TP to the collapsed distal bowel this mean partial obstruction, if there was minimal contrast in the distal loops this mean high grade, while if there was adequate contrast flows across the transition point this mean low grade. $(\mathbf{2 , 3 , 1 7 )}$

In the case of simple BO the blood flow continue in order, (4)on the other hand there are several serious complications of BO(perforation, ischemia, closed loop obstruction and Strangulation) threaten life in the case of delay diagnosis , $(\mathbf{1 , 3 , 4 )}$, in the current study only 8 cases reported complication 2 as ischemia and 6 as perforation . Each of this complication has different presentation in CT image which helped in diagnosing and differentiate between these medical condition, CT signs of strangulations are" poor or no contrast enhancement of bowel walls , target sign, mesenteric vasculature engorgement, and mesenteric congestion ", (5,18)while in closed-loop obstruction the signs are " distended bowel with radial mesenteric vessels with medial conversion ", in ischemia cases CT signs are "thickened intestinal walls and poor flow of contrast material into a section of bowel ",(1)and in necrosis and perforation, the main signs are " pneumatosis intestinalis, free intraperitoneal air, and mesenteric fat stranding ". (1)

However as all other radiology modalities there are few limitations of CT scan , (3,5)superficial lesions cannot precisely be seen, which make the diagnosis of low-grade or sub-acute obstructions difficult and need to be combine with contrast study particularenteroclysis $(\mathbf{5 , 1 9 )}$. Also the high dose of radiation is consider a big limitation in using CT scan specially between children, pregnant women and patients inflammatory bowel diseases (IBD) who need for recurrent imaging examination, however the new generation of multidetector CT (MDCT) scan combining with pediatric protocols showed significant influence in decreasing the radiation dose.(19,20,21,22,23)All of these limitations led the researcher to emphasize on the fact that CT scan is not a first line method to diagnose BO and it 
should use only in equivocal cases where there is no previous history of surgery or hernias and there is a high suspicion of complication. $(\mathbf{1 , 3})$

\section{Conclusion:-}

The early precise diagnosis of bowel obstruction helps in decreasing the morbidity and mortality rate. There are many radiology modalities help in the diagnosis where each modality has advantages and limitations . Plain abdomen radiography is suitable technique in the emergency cases and following after operation, however CT scan is the more accurate technique. Further studies need to be conducted to compare between these two technique and other modalities to provide more information to help doctors and radiologist in choosing the appropriate technique regarding the situation.

\section{References:-}

1. JACKSON PG and RAIJI M . Evaluation and Management of Intestinal Obstruction. Am Fam Physician. 2011 Jan 15;83(2):159-165.

2. Shakil $\mathrm{O}^{1}$, Zafar SN, Zia-ur-Rehman, Saleem S, Khan R, Pal KM .The role of computed tomography for identifying mechanical bowel obstruction in a Pakistani population. J Pak Med Assoc. 2011 Sep;61(9):871-4.

3. Manchanda SD ${ }^{1}$, Prasad A, Sachdev N, De P, Abbas SZ, Baruah BP. Multi detector computed tomography (MDCT) evaluation of small bowel obstruction: pictorial review. Trop Gastroenterol. 2010 Oct-Dec;31(4):249-59.

4. A NarishaMaharaj\&Bhugwan Singh (2015).A review of the radiological imaging modalities of non-traumatic small bowel obstruction, South African Family Practice, 57:3, 146-159, DOI: 10.1080/20786190.2014.977052

5. Jha AK . Radiological Techniques in the Diagnosis of Strangulating Small Bowel Obstruction. JNMA J Nepal Med Assoc. 2013 Apr-Jun;52(190):420-6.

6. Paulson EK, and Thompson WM. Review of Small-Bowel Obstruction: The Diagnosis and When to Worry. Radiology: May 2015. 275(2)

7. Maglinte DDT, Howard TJ, Lillemoe KD, et al. Small-bowel obstruction: State-of-the-art imaging and its role in clinical management. ClinGastroenterolHepatol. 2008;6(2):130-9. Available from: http://dx.doi. org/10.1016\%2Fj.cgh.2007.11.025

8. Thompson $\mathrm{WM}^{1}$, Kilani RK, Smith BB, Thomas J, Jaffe TA, Delong DM, Paulson EK. Accuracy of Abdominal Radiography in Acute Small-Bowel Obstruction: Does Reviewer Experience Matter?AJR Am J Roentgenol. 2007 Mar;188(3):W233-8.

9. Nicolaou S, Kai B, Ho S, et al. Imaging of acute small-bowel obstruction. Am J Roentgenol. 2005;185(4):1036-44. Available from: http://dx.doi. org/10.2214\%2FAJR.04.0815

10. Furukawa A, Yamasaki M, Takahashi M, et al. CT diagnosis of small bowel obstruction: scanning technique, interpretation and role in the diagnosis. Semin Ultrasound CT MRI. 2003, Oct;24(5):336-52. Available from: http://dx.doi.org/10.1016\%2Fj.sult.2003.08.001

11. Macari M, Megibow A. Imaging of suspected acute small bowel obstruction. SeminRoentgenol. 200;36(2):108-17.

12. Silva AC, Pimenta M, Guimaraes LS. Small bowel obstruction: whatto look for. Radiographics. 2009;29(2):423-9. Available from: http:// dx.doi.org/10.1148/rg.292085514

13. Algin $\mathrm{O}^{1}$, Evrimler S, Arslan H. Advances in Radiologic Evaluation of Small Bowel Diseases. J Comput Assist Tomogr. 2013 Nov-Dec;37(6):862-71.

14. Furukawa A., Yamasaki M., Furuichi K., Yokoyama K., Nagata T, Takahashi M, Murata K, Sakamoto T. Helical CT in the diagnosis of Small bowel obstruction. Radiographics. 2001;21 (2):341-55.

15. 19. Burkill GJC, Bell JRG, Healy JC. The utility of computed tomography in acute small bowel obstruction. ClinRadiol. 2001;56(5):350-9.

16. Jun L, ChangYi S . Diagnostic Value of Plain and Contrast Radiography, and Multi-slice Computed Tomography in Diagnosing Intestinal Obstruction in Different Locations. Indian J Surg. 2015 Dec;77(Suppl 3):1248-51.

17. Maglinte DD, Gage SN, Harmon BH, Kelvin FM, Hage JP, Chua GT, et al. Obstruction of the small intestine: accuracy and role of CT in diagnosis. Radiology. 1993;188:61-4.

18. Scaglione M, Romano S, Pinto F, Flagiello F, Farina R, Acampora C, Romano L. Helical CT diagnosis of small bowel obstruction in the acute clinical setting. Eur J Radiol. 2004;50(1):15-22.

19. Masselli G1, Gualdi G . CT and MR enterography in evaluating small bowel diseases: when to use which modality?Abdom Imaging. 2013 Apr;38(2):249-59.

20. Carucci LR, Levine MS (2002) Radiographic imaging of inflammatory bowel disease. GastroenterolClin North Am 31:93-117

21. 11. Podolsky DK (2002) Inflammatory bowel disease. N Engl J Med 347:417-429

22. 12. Maglinte DD, Sandrasegaran K, Lappas JC, Chiorean M (2007) CT enteroclysis. Radiology 245(3):661-671

23. 13. Fletcher JG, Fidler JL, Bruining DH, Huprich JE (2011) New concepts in intestinal imaging for inflammatory bowel diseases. Gastroenterology 140(6):1795-1806 